\title{
A PATTERN LANGUAGE FOR REUSING WEB TEACHING
}

\section{MATERIALS}

\author{
Nadia Bouz-Asal \\ Kyoto University \\ Yoshida-Honmachi, Sakyo-ku, Kyoto, Japan \\ nadia_bouzasal@yahoo.com \\ Rieko Inaba \\ Tsuda College \\ 2-1-1 Tsuda-machi, Kodaira-shi, Tokyo, Japan \\ inaba@tsuda.ac.jp \\ Toru Ishida \\ Kyoto University \\ Yoshida-Honmachi, Sakyo-ku, Kyoto, Japan \\ ishida@i.kyoto-u.ac.jp
}

\begin{abstract}
The Internet enables sharing of content between educators, which can reduce the time and effort needed to create custom teaching materials and improve the quality of students' learning experiences. There are, however, significant obstacles to effective educational content sharing and reuse, especially in developing countries. To address this problem, we have taken a user-centric approach to discover and analyze teachers' needs and to understand the main barriers to web content reuse among K-12 educators in Bosnia and Herzegovina. Based on this analysis, we have developed a novel pattern language for reusing web teaching materials.
\end{abstract}


Keywords: Web Content Sharing, Pattern Language, Education, E-Learning

\section{INTRODUCTION}

The Web has become the ultimate medium for information discovery and exchange, and provides access to a wealth of digital educational resources which are readily available in numerous subject areas. This abundance of educational resources has led to an increasing number of open educational content initiatives and an increase in the reuse and sharing of teaching materials all over the world. Teachers can now directly reuse materials and prepare new tailor-made materials by adapting existing ones, creating more effective learning experience for their students and improving the quality of their teaching practice. Current initiatives to further expand the culture of sharing and reusing Web educational materials have met with limited success. Several types of obstacles have been observed.

\section{Incompatibility and non-modifiability}

The presence of a wide range of e-learning products using different platforms can make content sharing and composition between e-learning systems difficult.

\section{Language}

A shortage of available educational resources in minority languages for the K-12 educational level, as well the absence of affordable technological infrastructures and trained staff, limits the ability of teachers in many developing countries to move forward on content reuse. The UNESCO's Universal Declaration on Cultural Diversity (2001) strives to decrease English language dominance on the Web by encouraging mother tongue teaching and promoting freedom of expression, media pluralism, multilingualism, and equal access to art, scientific and technological knowledge.

\section{Technology Focus}

The development of technology-push solutions, focused on resolving technological aspects such as system interoperability specifications and technical data and metadata standards, often fails to invest sufficiently in understanding teachers' real needs, and the behaviors and practices that are required for a successful adoption of educational content sharing solutions. 
The goal of the research reported here was to provide simpler and more effective processes for the reuse of Web teaching materials.

\section{METHOD -PATTERN LANGUAGES-}

A user-centric approach was adopted and combined with the pattern language approach. Pattern languages are a way to capture expert knowledge about solving recurring design problems in our environment. The pattern language approach originally emerged in architecture in the 1970's (Alexander, 1979) and has since gained momentum and acceptance across diverse fields and disciplines (Borchers, 2001), but has only recently emerged in the field of education and e-learning. Existing projects and initiatives in education using pattern languages focus mainly on solving problems within pedagogical and learning contexts, rather than problems in the development and reuse of educational materials.

The method of describing characteristic problem and solution traits is the usage of patterns. For the sake of clarity, comparability and readability, Alexander created a format to articulate patterns. The architect used his pattern language to define a new design planning process, taking the design of the University of Oregon as an example.

\section{PATTERN LANGUAGE AND PATTERN BASED WORKFLOW}

A Pattern Language consisting of 25 patterns was developed to capture and communicate practices and processes for reuse of Web teaching materials, and to provide a good starting place for future research, in both development of new patterns and development of designs for user-centric systems providing the functionalities needed for reusing Web teaching materials that were identified in the survey carried out among Bosnia-Herzegovina's K-12 teachers, to gain a deeper understanding of attitudes, practices and issues regarding existing educational resources on the Web. Figure 2 contains an example of an individual pattern, the Accuracy pattern, which is intended to guide the selection of material which is accurate and reliable for reuse.

\section{MODEL OF A MULTILINGUAL RESOURCE SYSTEM}

The aim of this research has been towards a bigger and more ambitious goal; to lay the foundation for building a multilingual learning resource system using Web service technologies which would enable educators to easily access and reuse unlimited number of educational resources. 
Being a multilingual resource system, it would contribute in reducing the imbalance of content availability in minority languages, especially for the K-12 educational level, as well as in further expanding the culture of sharing and reusing.

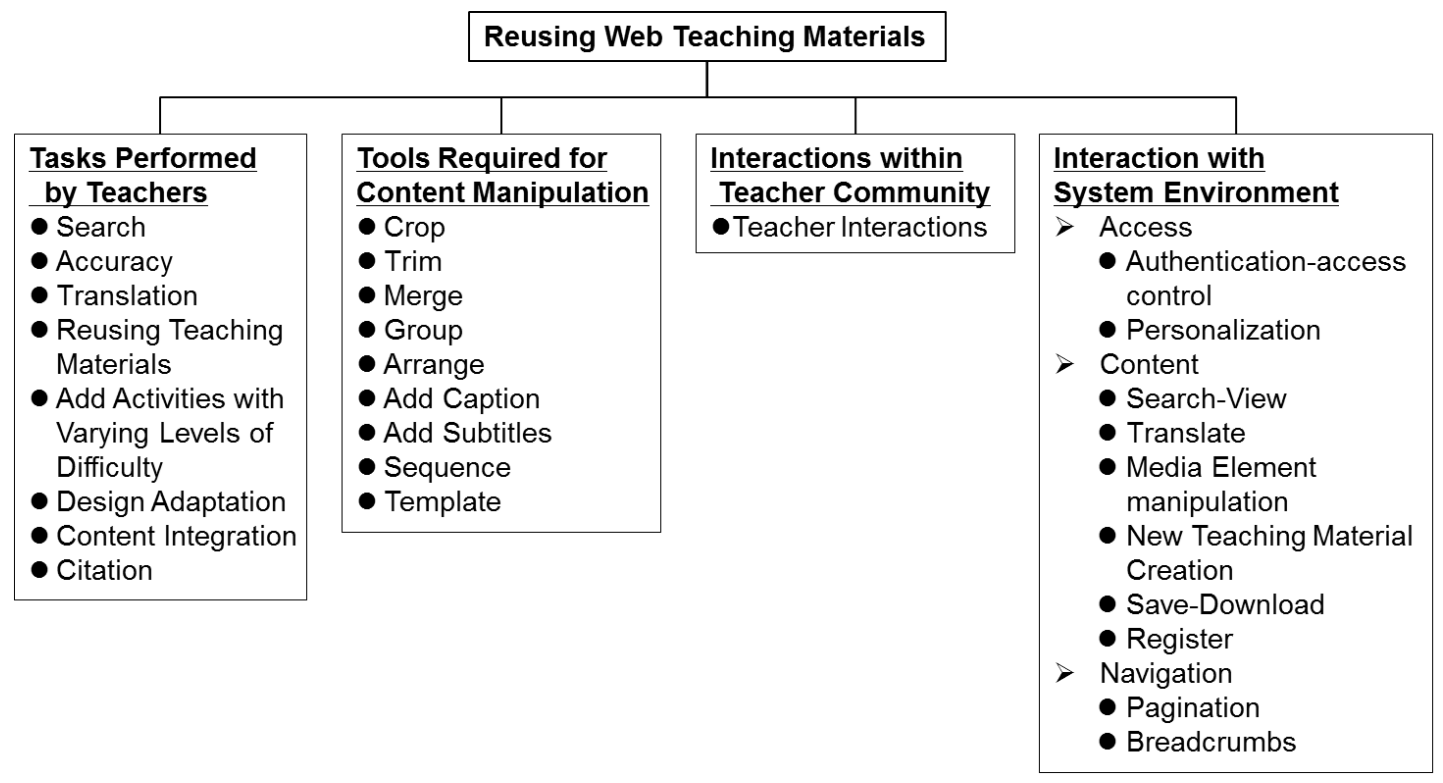

Figure 125 Patterns of a Pattern Language for Reusing Web Teaching Materials

A model of such a system supporting necessary user driven functionalities is illustrated in figure 3 . The system would take advantage of machine translators provided by the Language Grid project (Ishida, 2006) and collaboration tools provide by the Language Grid Toolbox to support the multilingual facet of resources and communication among the diverse teacher community. Each teaching material would be represented as a resource and wrapped as a service with different media element types and handling different levels of granularity, similar to that of the Language Grid. The user generated metadata level would allow users to add information about resources like topic, author, date of creation and editing, etc. As the amount of teaching materials would grow, a search function using classic keyword-based would not be enough or effective for efficient queries. Therefore, the search function would need the presence of content metadata formalized through various ontologies to refine search queries. 


\begin{tabular}{|l|l|}
\hline Accuracy & Content accuracy of teaching materials found on the Web \\
\hline Intent & Determine content accuracy of existing teaching materials on the Web \\
\hline Context & $\begin{array}{l}\text { The Web offers a wide range of information that can be easily accessed and downloaded, however anyone } \\
\text { can post content and there is no quality control. Educators need to check the accuracy of teaching material } \\
\text { content. }\end{array}$ \\
\hline Problem & How to determine accuracy and reliability of teaching materials? \\
\hline Forces & $\begin{array}{l}\text { - Teaching content is provided by unknown and possibly unreliable sources } \\
\text { - Need to distinguish presence of political, cultural, religious or personal biases }\end{array}$ \\
\hline Solution & $\begin{array}{l}\text { No quality assurance for information on the Web exists. Generally, information created by government } \\
\text { (.gov) and educational institutions (.edu) is considered the most reliable. Before deciding to reuse content, } \\
\text { teachers must critically inspect whether information is accurate, comprehensive, credible and objective. } \\
\text { The following steps will help teachers decide whether to reuse web content. }\end{array}$ \\
$\begin{array}{l}\text { 1. Identify the author. Reliable materials should state the author's name. } \\
\text { 2. Identify contact information. Reliable materials should provide a way to contact authors to ask questions } \\
\text { or debate about information in the materials. }\end{array}$ \\
$\begin{array}{l}\text { 3. Identify author's purpose or motivation for the material and assess its objectivity. Reliable sources } \\
\text { should present information objectively. }\end{array}$ \\
\begin{tabular}{|l} 
4. Identify sources of any facts mentioned. Reliable materials should reference published sources of facts \\
found in them so that they can be checked.
\end{tabular} \\
\begin{tabular}{|l} 
5. Identify any links to related sources of information on the covered topics. Information in reliable \\
materials can be verified against other sources. \\
q. Identify the date of last modification. Reliable contents should be up-to-date, though this may vary \\
according to the subject and type of educational field.
\end{tabular} \\
\hline Related & $\begin{array}{l}\text { Accurate information content is provided to students in teaching materials. } \\
\text { Teaching materials must be updated to reflect changes in covered concepts. }\end{array}$ \\
\hline \begin{tabular}{l} 
Search, Translation, and Reusing Web Teaching Materials patterns \\
\hline
\end{tabular}
\end{tabular}

Figure 2 The Accuracy Pattern for Selecting Web Teaching Material

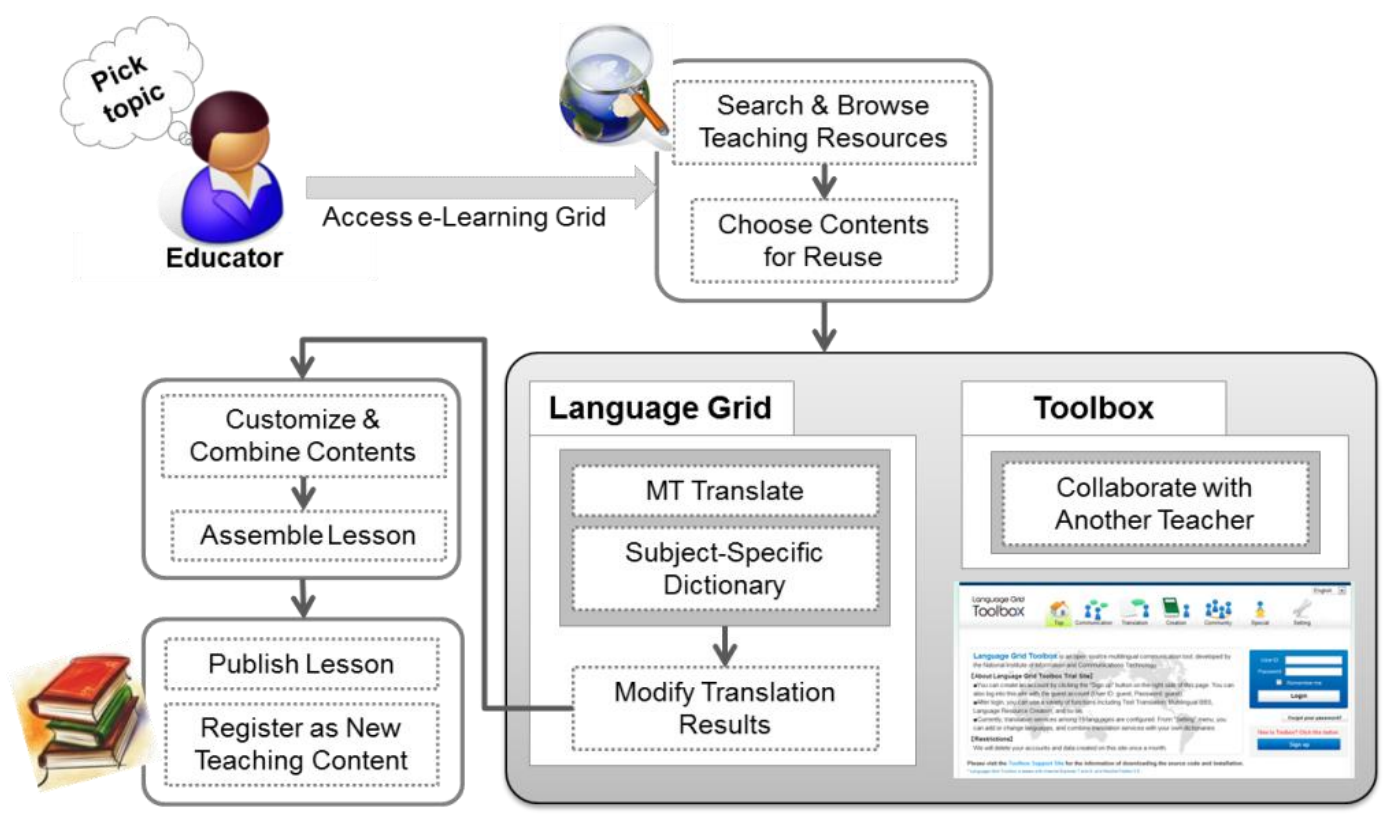

Figure3 Model of a Multilingual Resource System 


\section{CONCLUSIONS}

A logical solution to the problem of creating custom teaching materials to improve the quality of students' learning experiences within reasonable bounds on time and effort lies in taking advantage of information and communication technologies and the vast amounts of educational resources readily available on the Web. In order to supplement the user-centric approach, this paper has also adopted the pattern language approach, adopted by so many other diverse fields and domains over the years, as a proven method to share expertise knowledge and solve real world problems. A pattern language consisting of 25 patterns which addresses different aspects necessary for successful reuse of Web teaching materials like tasks, tools, and system and community interactions was developed.

\section{ACKNOWLEDGEMENT}

We are grateful for the assistance of David Kinny. This research was partially supported by a Grant-in-Aid for Young Scientists (B) $(24700115,2012-2013)$ from the Japan Society for the Promotion of Science (JSPS).

\section{REFERENCES}

Alexander, C. (1979). The Timeless Way of Building. Oxford University Press.

Borchers, J.O. (2001). A pattern approach to interaction design. AI \& SOCIETY, 15(4), 359-376. http://dx.doi.org/10.1007/BF01206115.

Ishida, T. (2006). Language grid: An infrastructure for intercultural collaboration. In B. Werner (Ed.), Proceedings of the International Symposium on Applications on Internet (pp. 96-100). Phoenix, USA: IEEE Computer Society.

UNESCO. (2001). Retrieved October 2, 2013, from http://portal.unesco.org/en/ev.php-URL_ID=13179\&URL_DO=DO_TOPIC\&URL_ SECTION=201.html. 